\title{
Hybrid tracking detector based on semiconductor and gas technologies
}

\author{
George Bashindzhagyan ${ }^{1}$, Natalia Korotkova $^{1, *}$, Anatoli Romaniouk $^{2}$, Nikolai $_{\text {Sinev }}{ }^{3}$, \\ Vladimir Tikhomirov ${ }^{2 ; 4}$ \\ ${ }^{1}$ M.V.Lomonosov Moscow State University, Russia \\ ${ }^{2}$ National Research Nuclear University MEPhI, Russia \\ ${ }^{3}$ University of Oregon, Eugene, USA \\ ${ }^{4}$ P. N. Lebedev Physical Institute of the Russian Academy of Sciences, Russia
}

\begin{abstract}
New Silicon-Gas Pixel Detector (SiGPD) is the result of the further development already proposed Gas-Pixel Detector (GPD). The only disadvantage of GPD was the uncertainty of the particle arriving time. The problem can be solved by additional silicon pixels implementation inside existed electronic chip epitaxial layer during regular chip production. The cost of new Si-Gas Pixel Detector remains practically the same. The new detectors have self-triggering properties and can be used for a first level trigger generation in the particle physics experiments and for many other applications.
\end{abstract}

\section{Gas Pixel Detector - the basis of new detector}

Gas Pixel Detector (GPD) [1] is a small flat drift chamber with $10 \mathrm{~mm}$ thick gas volume and with a large electronic chip with pixel sensors about $100 \times 200 \mu \mathrm{m} 2$ as anode plane. A mesh is placed in about $50 \mu \mathrm{m}$ in front of the sensors. Strong electric field between the mesh and anode sensors $(\sim 100 \mathrm{kV} / \mathrm{cm})$ allows to obtain gas amplification of the order of $10^{3}$ to register every electron crossing the mesh.

GPD has the following advantages:

- Very low material and relatively cheap structure is combined with good particle track reconstruction.

- Time-projection method application and specially developed algorithms allow 3D particle track segment reconstruction with position accuracy of $\sim 30 \mu \mathrm{m}$ and angle measurement precision better than $0.5^{\circ}$.

- Because particle track reconstruction is realized on hardware level within chip electronics, it can be done very fast in about $1 \mu$ s if $25 \mathrm{~ns}$ readout cycle is used. This performance allows GPD to be used for a first level trigger generation in accelerator experiments.

* Corresponding author: korona@mail.cern.ch 


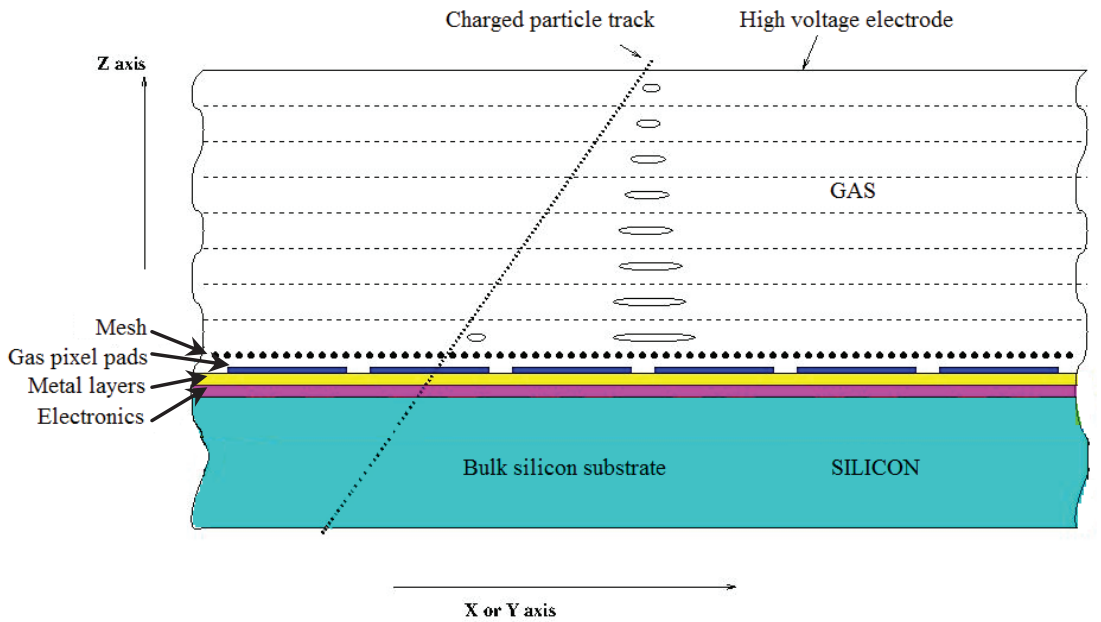

Fig. 1. The schematic view of GPD structure.

\section{Time-projection readout}

Electrons generated by an ionizing particle in gas drift transversely towards the chip surface, where they are registered by the pixels of the chip every readout cycle.

The process of transverse diffusion spreads electrons during their drift, and up to 50 pixels can be fired during one readout cycle $(25 \mathrm{~ns})$. This improves the accuracy of the measurements with relatively large pixels, but creates a problem to transmit and analyze the data online.

With $25 \mathrm{~ns}$ readout cycle, $10 \mathrm{~mm}$ thick gas volume and maximum drift time of electrons about 200 ns, we can obtain 8 points with $\mathrm{X}$ and $\mathrm{Y}$ coordinates along the particle track and $\mathrm{Z}$ coordinate, which corresponds the number of the cycle when the coordinates were measured (figure 2).
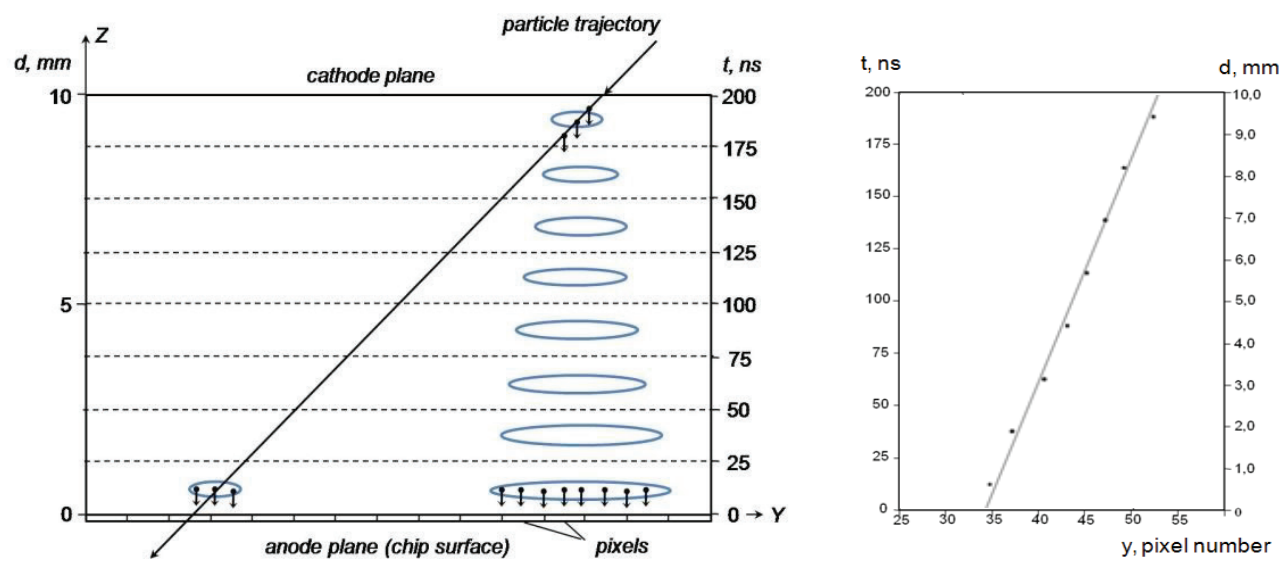

Fig. 2. Electrons collecting on the GPD chip plane (left). The example of the particle trajectory reconstruction (right). 


\section{Online track reconstruction algorithm}

Figure 3 presents a typical cluster structure in XY plane and its projections on both axes for the 3 -rd of 8 cycles. To calculate the particle position we use center of mass approach. In each projection the number of the row $\left(n_{X}^{i}\right)$, is multiplied by the number of fired pixels in this row $\left(R_{x}^{i}\right)$, and the sum of the products is divided by the total number of fired pixels in the cluster $N_{P}: X=\sum R_{X}^{i} n_{X}^{i} / N_{P}, Y=\sum R_{Y}^{j} n_{Y}^{j} / N_{P}$.

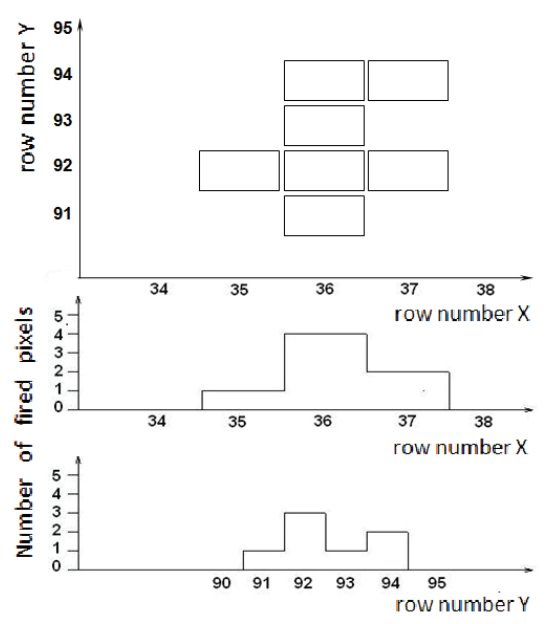

Fig. 3. The example of the cluster structure in $\mathrm{XY}$ plane and its projections on $\mathrm{X}$ and $\mathrm{Y}$ axes.

In experiments operating at high trigger rate the basic data processing must be performed already on chip level. Every pixel has an integrated fast preamplifier, discriminator and flip-flop with two fixed current outputs connected to common $\mathrm{X}$ and $\mathrm{Y}$ lines going along the particular $X_{i}$ and $Y_{j}$ rows correspondingly (figure 4). If a pixel is fired during a cycle, FF turns $\mathrm{ON}$ and current flows along the line.

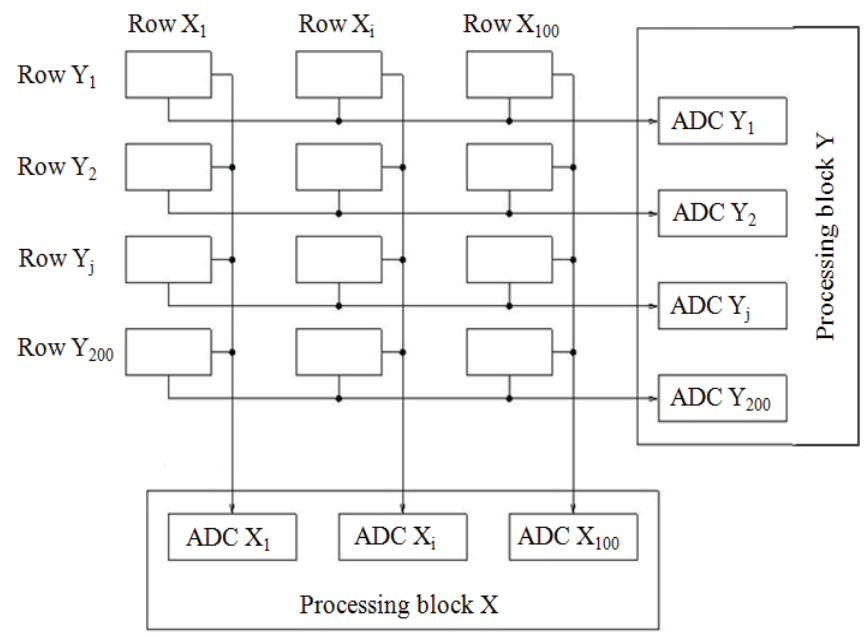

Fig. 4. The block diagram of the readout algorithm. 


\section{Expected and measured resolution}

To estimate the expected resolution of GPD MC simulations for different detector geometries and gas mixtures have been done.

The examples of simulated distributions for $20 \mathrm{GeV}$ pions crossing the detector with $100 \times 200 \mu \mathrm{m}^{2}$ pixels and Xe gas mixture at angles $\theta=20^{\circ}$ and $\varphi=3^{\circ}$ are shown in figure 5 .
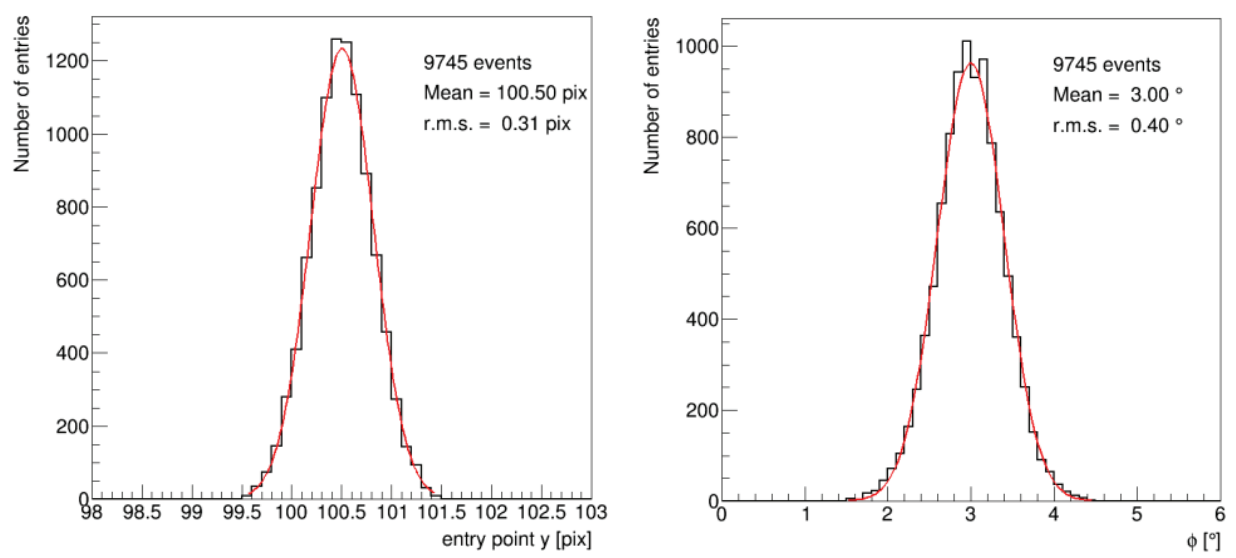

Fig. 5. The distribution of the reconstructed track entry points $Y$ coordinates (left) and incident angles $\varphi$ (right) for $20 \mathrm{GeV}$ pions crossing the GPD with $\theta=20^{\circ}$ and $\varphi=3.00^{\circ}$.

Performance of GPD detector with DME/CO2 mixture and $55 \times 55 \mu \mathrm{m}^{2}$ pixels for particles crossing the detector plane the angle $30^{\circ}$ is shown in figure 6 [2].
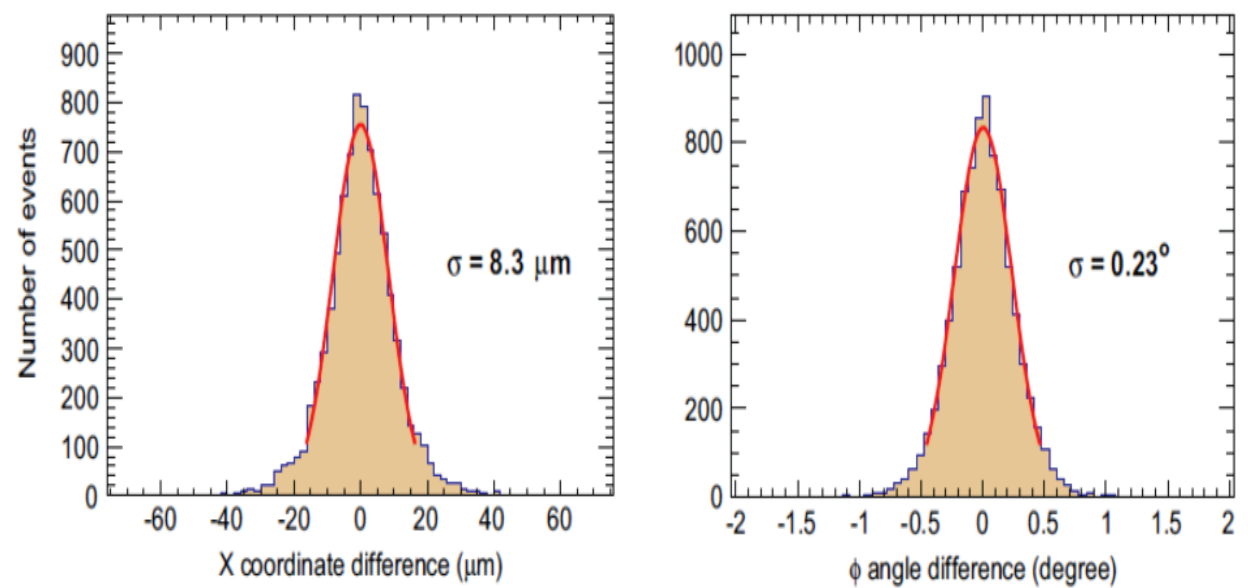

Fig. 6. Position determination accuracy of GPD detector with DME/CO2 mixture and $55 \times 55 \mu \mathrm{m}^{2}$ pixels across the track projection (right plot) and angle measurement accuracy of the track projection on the chip plane (left plot). Solid lines represent Gaussian fits applied to the central parts of distributions. 


\section{Silicon pixels Implementation into GPD electronic chip}

GPD assumes permanent data collection and processing with cycles corresponding collider beam crossing cycle. The disadvantage of the GPD is the absence of exact time knowledge when the particle crossed the detector. It becomes more essential if a few particles arrived during $200 \mathrm{~ns}$ drift time needed to collect the data.

The simple way to obtain time coordinate is to add a layer of silicon pad or strip detector to existed GPD structure. But it means additional layer of silicon, a lot of interconnections and essential increase of production cost. Therefore we propose to use charged particle detection in epitaxial layer created during regular chip production technology.

Adding silicon sensors below chip electronics within epitaxial layer we can collect electrons generated by the charged particle in the epitaxial layer $(\sim 7-10 \mu \mathrm{m})$ in very short time about $1 \mathrm{~ns}$ and obtain exact information about the particle arrival very soon. Despite the charge generated in epitaxial layer is about $700 \mathrm{e}$, the signal at the amplifier input is sufficient for its registration because of very low capacitance of the silicon pixel $(\sim 1 \mathrm{pF})$.

\section{Silicon-Gas Pixel Detector}

Silicon-Gas Pixel Detector (SiGPD) [3] is a further development of the GPD concept with the determination of the exact time when particle crossed the detector, which can be realized by implementation of silicon pixel layer inside electronic chip without other changes in the existing GPD design and therefore without increasing the production cost of the device.

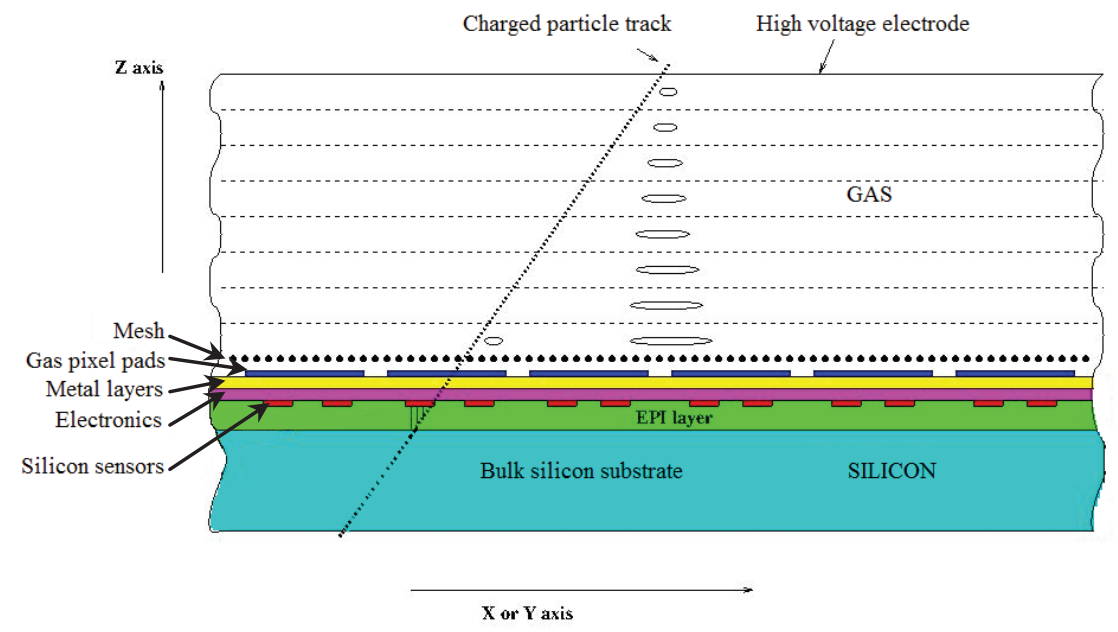

Fig. 7. The schematic view of the SiGPD structure.

In addition to the GPD features SiGPD have the following properties and advantages.

- With implemented silicon pixel layer we exactly know the time when particle crossed the detector and can use SiGPD as a completely self-triggered device.

- SiGPD can be used to generate fast trigger in accelerator experiments.

- SiGPD has an internal generator which starts readout process when particle crossed detector volume. If SiGPD works in accelerator environment its clocks must be synchronized with beam crossings. Suggested electronics can generate output signal for use in the level 1 trigger system. The latency of the output data is about 40 clock periods. With $40 \mathrm{MHz}$ frequency used in LHC, it is $1 \mu \mathrm{s}$. If smaller latency is required one can 
increase frequency to $80 \mathrm{MHz}$ and decrease latency to about $0.5 \mu \mathrm{s}$. If $0.25 \mu \mathrm{m}$ technology is used for electronics production, $80 \mathrm{MHz}$ looks like reasonable clock speed.

- Silicon pixels in SiGPD does not require high-resistivity silicon and can be implemented into GPD electronic chip within the standard chip production technology without increasing the production cost.

- All the silicon and gas pixel electronics, which measures and analyses signals from $\mathrm{X}$ and Y lines, is fully integrated in electronic chip structure and produced within the same chip production process. This means that SiGPD does not require any external electronics for its operation.

- SiGPD have higher radiation hardness compare to regular silicon detector, because of its very thin epitaxial detecting layer.

\section{SiGPD readout}

As it was described earlier for GPD signals from gas pixels in each row $X_{i}$ (or $Y_{j}$ ) are added together and transmitted to $\mathrm{X}$ (or $\mathrm{Y}$ ) coordinate processing block, situated at the edges of the electronic chip. We may compare this readout system with two microstrip detectors with perpendicularly going strips. It seriously simplified and speeds up readout procedure. If particle density is high and a few particles can appear at the same clock we can avoid ambiguity adding one more interconnecting system of wires.

Readout from Si sensors operates similar way as for the gas pixels, and even easier because it does not require a number of fired pixels in each row. In other words, we apply "binary" readout for silicon and "analogue" for gas pixels.

With binary silicon pixel readout we can determine XY coordinate of the particle arrival with $7 \mu \mathrm{m}$ accuracy. If power consumption/dissipation is a critical point, the silicon pixel size can be increased to $50 \times 50 \mu \mathrm{m}$. This case $\mathrm{XY}$ accuracy is still $15 \mu \mathrm{m}$ but power dissipation is four times less.

Chip electronics consists of blocks under each gas pixel pad, peripheral X \& Y coordinate processing blocks and some communication logic to transfer data to host computer. The layout of SiGPD chip is shown schematically in figure 8.

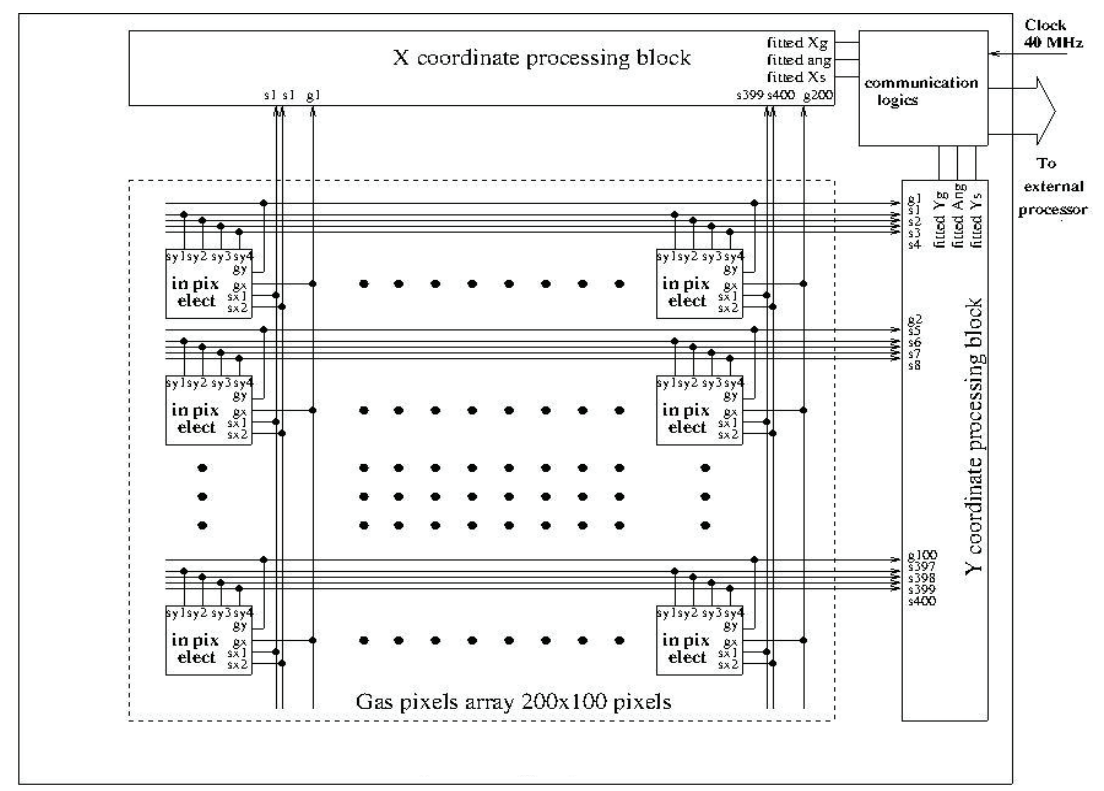

Fig. 8. SiGPD chip layout. 


\section{Conclusion}

A concept of new Silicon-Gas Pixel Detector is developed. This new detector is based on proposed earlier Gas Pixel Detector. But with implemented into existing electronic chip silicon pixels new detector has significantly extended functionality and becomes more convenient and universal device.

Fast online data processing and ability to present full information about charged particle trajectory in $\sim 0.5 \mu$ s can be very useful for a fast trigger generation.

Very low material $(\sim 10 \mathrm{~mm}$ gas and $\sim 100 \mu \mathrm{m} \mathrm{Si})$ and relatively cheap structure are combined with good coordinate $(\sim 5-10 \mu \mathrm{m})$ and angular $\left(\sim 0.4^{\circ}\right)$ accuracies of particle track segment reconstruction.

SiGPD works as a completely self-triggered device and can be used in many areas, where particle trajectory reconstruction is required.

This work was partially supported by Russian Foundation for Basic Research (Grant 14-22-0353).

\section{References}

1. N. Sinev, G. Bashindzhagyan, N. Korotkova, A. Romaniouk, V. Tikhomirov. Journal of Physics: Conference Series, 675 (2016)

2. A. Boldyrev, F. Hartjes, N. Hessey, M. Fransen, S. Konovalovet et al., Nucl. Instr. and Meth. A 807 (2016)

3. N. Sinev, G. Bashindzhagyan, N. Korotkova, A. Romaniouk, V. Tikhomirov. Journal of Physics: Conference Series, 798 (2017) 